\section{Response to: 'Does the prevalence of radiographic hand osteoarthritis in patients with HIV-1 infection increase or not?' by Luo et al}

We thank Luo et $a l^{1}$ for their interest in our work demonstrating for the first time that patients with HIV-1 infection display radiographic hand osteoarthritis (HOA) more frequently than the general population and that metabolic syndrome (MetS) is an independent factor associated with HOA presence and severity in the setting of HIV-1 infection. ${ }^{2}$ We would like to respond point-to-point to each comment.

Not all confounding factors, such as genetic factors, have been taken into account when we compared the prevalence radiographic HOA between the Framingham and the Metabolic Syndrome and Fibrosis-Osteoarthritis (METAFIB-OA) cohorts as well as between patients with HIV-1 infection w/o MetS in the METAFIB-OA cohort. However, a major purpose of our study was to compare the prevalence of HOA in patients who had HIV-1 infection with that from the Framingham cohort. The Framingham Study has been used as a group representative of the general population in many previous studies including in recent lupus studies. ${ }^{3}{ }^{4}$ Moreover, the comparison between the two groups (w/o MetS) within the METAFIB-OA cohort took into account the main risk factors of HOA previously identified such as age, sex and previous joint injury. Since sex and age have a strong impact on HOA occurrence, we chose to stratify all the analyses according to these two parameters. The other factors mentioned by Luo et al, such as atherosclerosis, bone mineral density, life-style factors, are not considered as strong risk factors for osteoarthritis (OA) in the hand but only associated with HOA in some epidemiological studies. ${ }^{5}$ Thus, we think that they are not relevant to be used as adjusting factors in our study which focused on MetS and risk of HOA.

We agree with the authors that the Framingham cohort is from the USA, which is possibly quite different from the METAFIB-OA cohort. ${ }^{6}$ However, age and sex being certainly the two strongest risk factors of HOA, we stratified our crude comparison according to these factors, although we acknowledge that some other risk factors may have been omitted.

We measured several blood biomarkers in order to explain the association between MetS and HOA and to determine whether some HIV characteristics are associated with HOA (since all patients in METAFIB are HIV-1-infected). These biological assessments were not performed to find markers for HOA diagnosis. Interestingly, plasma sCD14 level, that reflects macrophage activation, was associated with HOA but this association did not persist after adjustment on MetS. This result does not raise concerns about association between HOA and HIV-1 since all patients tested were HIV-1-infected but emphasises the role of macrophage activation, a well-known surrogate marker of MetS-induced systemic low-grade inflammation, as one of the possible pathways linking MetS and HOA. We did not consider HIV-1 infection as a determinant of HOA. But based on such a cross-sectional study, we suggest that this association was related to ageing and MetS.

One of the strengths of our study is to demonstrate, in the special setting of HIV-1 infection, that MetS increases radiographic HOA prevalence and severity. The two references (ie, 9 and 10) cited by Luo et al did not report any association between HOA and MetS. Conversely, Dahaghin et al previously showed that accumulation of overweight, diabetes and hypertension was associated with an increased risk of $\mathrm{HOA},{ }^{7}$ but without using international criteria of $\mathrm{MetS}^{8}$ and extensive adjustments, as we did.

The METAFIB-OA cohort is mainly composed of men, due to the sex ratio of HIV-1 infection in developed countries. The comparison of HOA prevalence between women from Framingham and METAFIB-OA cohorts and between patients with HIV-1 infection w/o MetS gave almost similar results as the analyses performed in men, but did not reach significance for all analyses, perhaps due to the smaller number of women. Furthermore, the differences observed in men strengthen our results since analyses stratified by sex avoided the impact of gender which is a strong risk factor for HOA.

Luo et al raised concerns about hand radiographic scoring. However, a kappa coefficient above 0.60 for the Kellgren-Lawrence scale is considered good, not low and the other criteria (intraclass correlation coefficient above 0.90 ) was excellent. Additionally, one of the readers has previously confirmed her reliability with another expert in the reading of hand radiographs. ${ }^{9}$ Moreover, our definition of radiographic HOA was more stringent than that used in Framingham ${ }^{6}$ and thus we may have actually underestimated the difference of prevalence of HOA between populations.

Therefore, we confirm that the prevalence of HOA in our cohort of HIV-infected individuals was more frequent than in a control population and linked to the presence of MetS, which is at present frequently observed in ageing HIV-infected individuals. ${ }^{10}$

\section{Jérémie Sellam, ${ }^{1,2}$ Karine Lacombe, ${ }^{3,4}$ Anne-Laurence Tomi, ${ }^{5}$ Ida K Haugen, ${ }^{6}$ David T Felson, ${ }^{7,8,9}$ Soraya Fellahi, ${ }^{2,8}$ Jean-Philippe Bastard ${ }^{2,8}$ Anne Miquel, ${ }^{9}$ Manuela Sebire, ${ }^{4}$ Caroline Rey-Jouvin, ${ }^{1}$ Emmanuel Maheu, ${ }^{1}$ Jacqueline Capeau, ${ }^{2,8}$ Pierre-Marie Girard, ${ }^{3,4}$ Jean-Luc Meynard, ${ }^{3}$ Francis Berenbaum ${ }^{1,2}$ \\ ${ }^{1}$ Department of Rheumatology, DHU i2B, Saint-Antoine Hospital, Assistance Publique-Hôpitaux de Paris (AP-HP), Paris, France \\ ${ }^{2}$ Sorbonnes Universités, UPMC Univ Paris 06, Inserm UMRS_938, DHU i2B, Paris, France \\ ${ }^{3}$ Sorbonnes Universités, UPMC Univ Paris 06, INSERM, Institut Pierre Louis d'épidémiologie et de Santé Publique (IPLESP UMRS 1136), Paris, France ${ }^{4}$ Department of Infectious Diseases, Saint-Antoine Hospital, AP-HP, Paris, France \\ ${ }^{5}$ Department of Rheumatology, Henri Mondor Hospital, AP-HP, Créteil, France \\ ${ }^{6}$ Department of Rheumatology, Diakonhjemmet Hospital, Oslo, Norway \\ ${ }^{7}$ Clinical Epidemiology Unit, Boston University School of Medicine, Boston, Massachusetts, USA \\ ${ }^{8}$ Department of Biochemistry, Tenon Hospital, AP-HP, Sorbonnes Universités, UPMC Univ Paris 06, Inserm UMR_S938, DHU i2B, IHU ICAN, Paris, France \\ ${ }^{9}$ Department of Radiology, Saint-Antoine Hospital, AP-HP, Paris, France}

Correspondence to Professor Francis Berenbaum, Saint-Antoine Hospital, Department of Rheumatology, 184 rue du Faubourg Saint-Antoine, Paris 75012 , France; francis.berenbaum@aphp.fr

Competing interests None declared.

Provenance and peer review Commissioned; internally peer reviewed.

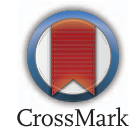

To cite Sellam J, Lacombe K, Tomi A-L, et al. Ann Rheum Dis 2016;75:e52.

Received 20 May 2016

Accepted 27 May 2016

Published Online First 21 June 2016

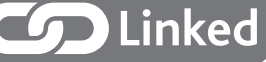

- http://dx.doi.org/10.1136/annrheumdis-2016-209808

Ann Rheum Dis 2016;75:e52. doi:10.1136/annrheumdis-2016-209833 


\section{REFERENCES}

1 Luo W, Lei G, He $H$, et al. Does the prevalence of radiographic hand osteoarthritis in patients with HIV-1 infection increase or not? Ann Rheum Dis 2016;75:e51.

2 Tomi AL, Sellam J, Lacombe K, et al. Increased prevalence and severity of radiographic hand osteoarthritis in patients with HIV-1 infection associated with metabolic syndrome: data from the cross-sectional METAFIB-OA study. Ann Rheum Dis 2016; Published Online First 31 Mar 2016. doi:10.1136/annrheumdis-2016209262

3 Karp I, Abrahamowicz M, Fortin PR, et al. Longitudinal evolution of risk of coronary heart disease in systemic lupus erythematosus. J Rheumatol 2012; 39:968-73.

4 Manzi S, Meilahn EN, Rairie JE, et al. Age-specific incidence rates of myocardial infarction and angina in women with systemic lupus erythematosus: comparison with the Framingham Study. Am J Epidemiol 1997;145:408-15.

5 Kloppenburg M, Kwok WY. Hand osteoarthritis--a heterogeneous disorder. Nat Rev Rheumatol 2011;8:22-31.
6 Haugen IK, Englund M, Aliabadi P, et al. Prevalence, incidence and progression of hand osteoarthritis in the general population: the Framingham Osteoarthritis Study. Ann Rheum Dis 2011;70:1581-6.

7 Dahaghin S, Bierma-Zeinstra SM, Koes BW, et al. Do metabolic factors add to the effect of overweight on hand osteoarthritis? The Rotterdam Study. Ann Rheum Dis 2007;66:916-20

8 Alberti KGMM, Eckel RH, Grundy SM, et al. Harmonizing the Metabolic Syndrome: a Joint Interim Statement of the International Diabetes Federation Task Force on Epidemiology and Prevention; National Heart, Lung, and Blood Institute; American Heart Association; World Heart Federation; International Atherosclerosis Society; and International Association for the Study of Obesity. Circulation 2009;120:1640-5.

9 Bijsterbosch J, Haugen IK, Malines C, et al. Reliability, sensitivity to change and feasibility of three radiographic scoring methods for hand osteoarthritis. Ann Rheum Dis 2011;70:1465-7.

10 Alencastro PR, Wolff FH, Oliveira RR, et al. Metabolic syndrome and population attributable risk among HIVIAIDS patients: comparison between NCEP-ATPIII, IDF and AHA/NHLBI definitions. AIDS Res Ther 2012;9:29. 